\title{
APPLICATIONS OF CUTTING STOCK PROBLEM
}

We present one heuristic solution for the well-known cutting stock problem which was formulated by Kantorovich in 1939. It is the problem of filling an order at minimum cost for specified numbers of lengths of material to be cut to given stock lengths of given cost. When expressed as an integer programming problem the large number of variables involved generally makes computation infeasible. The same difficulty persists when only an approximate solution is being sought by linear programming.

Keywords: model, linear programming, cutting stock, optimal solution, lower bound.

\section{Introduction}

The first known formulation of cutting stock problem was given in 1939 by the Russian economist and mathematician Kantorovich known to be NP complete problem [1]. The primary reasons for this activity are that cutting stock problems occur in a wide variety of industrial applications and there is a large economic incentive to find more effective solution procedures. It is easy to compare alternative solution procedures and to identify the potential benefits of using a proposed procedure [2].

For example imagine that you work in a factory and you have a number of valuable rod stocks waiting to be cut, yet different customers want different numbers of rods of various-sized lengths (e. g. for steel railway bridge [3]). How are you going to cut the rod stocks so that you minimize the waste?

\section{Formulation of the problem}

Let $R_{i}$ be the nominal order requirements for rods of length $s_{i}, s_{i} \neq s_{j}$ for any elements $i, j, i \neq j i=1, \ldots, m, j=1, \ldots, m$, to be cut from rod stocks of usable length $s, b_{i}$ is the lower bound on the order requirement for rods of length si and $c_{j}$ is the cost of pattern $j$ [2]. This problem can be formulated as the following model:

$$
\begin{aligned}
& \text { Minimize } \sum_{j=1}^{n} c_{j} x_{j} \\
& \text { subject to } \sum_{j=1}^{n} a_{i j} x_{j} \geq b_{i} i=1, \ldots, m, \\
& \text { where } x_{j} \geq 0 \text { and } x_{j} \text { are integer, }
\end{aligned}
$$

$a_{i j}$ is the number of rods of length $s_{i}$ to be slit from each rod stock that is processed using pattern $j$. In order for the elements $a_{i j}$, $i=1, \ldots, m$, to constitute a feasible cutting pattern, the following restriction must be satisfied:

$$
\sum_{j=1}^{m} a_{i j} s_{i} \leq s,
$$

where $a_{i j} \geq 0$ and $a_{i j}$ are integer,

$x_{j}$ is the number of rod stocks to be slit using pattern $j$, and $c_{j}$ is the trim loss incurred by pattern $j, c_{j}=s-\sum_{i=1}^{m} a_{i j} s_{i}$.

\section{Solution approach}

The main idea of solution approach lies in a scheduling of cutting stock in descending order with respect to a length of rods.

For this reason we sort out a set $S$ of all order requirements $s_{i}$ in descending order that yields the set $S=\left\{s_{1}, \ldots, s_{n}\right\}$, where $s_{i} \geq$ $\geq s_{i+1}$ for $i=1, \ldots, n-1$. It seems that $m \leq n$ with respect to items $b_{i}$, where $b_{i} \geq 1$ and $b_{i}$ are integer. Especially if any $b_{i}=1$, $i=1, \ldots, m$ then $m=n$.

\section{Procedure}

1-st step. We create the set $S_{k}=\left[s_{1}^{*}, \ldots, s_{k}^{*}\right]$, where $s_{1}^{*}, \ldots, s_{k}^{*}$ are the first $k$ possible requirements of the set $S$ such that $s_{1}^{*}+\ldots+$ $+s_{k}^{*}+r_{k}=s$ and $r_{k}<s_{i}$ for $i=1, \ldots, n$. Here $r_{k}$ represents a remainder by scheduling of cuts. Obviously this remainder is less than the arbitrary requirement $s_{i} \in S$.

2-nd step. We will progressively remove items of greatest lengths from the set $S_{k}$ and add the nearest shorter items to $S_{k}$ from the $S$. By this way we will find a nearest $s_{i}^{*}$ greater than $s_{i+1}^{*}$

\footnotetext{
* Vladimir Medvid

Department of Mathematics, Faculty of Humanities, University of Zilina, Slovakia, E-mail: vladimir.medvid@fhv.uniza.sk
} 
in the set $S_{k}$. The item $s_{i}^{*}$ will be removed from $S_{k}$ and added the nearest shorter items of the lengths $s_{i+1}^{*}, \ldots, s_{i+l}^{*}$ to $S_{k}$ until the condition is satisfied

$s-\left(s_{1}^{*}+\ldots+s_{i-1}^{*}+s_{i+1}^{*}+\ldots+s_{i+l}^{*}\right)=r_{k}$, where $r_{k}<s_{i}, s_{i} \in$ $\in S$ for $i=1, \ldots, n$ and $S_{k}=\left\{s_{1}^{*}, \ldots s_{i-1}^{*}, s_{i+1}^{*}, \ldots, s_{i+1}^{*}\right\}, l$ is positive integer.

We repeat this step until the order set $S$ has the same items of the greatest lengths. Finally we state as the 1-st cut of the stock We remove all items $s_{j} \in S_{k}$ from the set $S$, i. e. $S:=S-S_{k}$ and return on the 1-st step and continue. (a command $A:=B$ means that the content of the set $B$ is moved into the set $A$ ). We will repeat this procedure until the set $S$ is empty.

\section{Algorithm}

Let $S=\left\{s_{1}, s_{2}, \ldots, s_{n}\right\}, S$ is the set of the rods of the lenghts $s_{l}$, where $s_{l} \geq s_{l+1}, l=1,2, \ldots, n, s$ is a size of a whole rod stock, $R$ is a set of remainders, $S_{c}=\emptyset$.

$$
k=0
$$

0. $k$ : $=k+1$. Let $R=\emptyset, S^{*}=\emptyset$. Let $b$ be a number of rods of a maximal length $s_{1}$ and $S_{k}^{b}=\emptyset$. Choose the first $j$ nearest possible rods of the lengths $s_{1}^{*}, s_{2}^{*}, \ldots, s_{j}^{*}$ from the set $S$ such that $s_{1}^{*}$, $s_{2}^{*}, \ldots, s_{j}^{*}+r_{b+1}=s$, where $r_{b+1}<s_{l}$ for $l=1,2, \ldots, n$ ( $l$ (iota) is the letter of the Greek alphabet). If $r_{b+1}=0$ then $S_{c}:=S_{c} \cup\left\{S_{k}^{b}\right\}$ and go to 3 .

$$
\text { Put } S_{k}^{b}=\left\{s_{1}^{*}, s_{2}^{*}, \ldots, s_{j}^{*}\right], R:=R \cup\left\{r_{b+1}\right\}
$$

1. If there are the nearest rods of the lengths $s_{i}^{*}, s_{i+1}^{*} \in S_{k}^{b}$ such that $s_{i}^{*}>s_{i+1}^{*}$ then subtract $s_{i}^{*}$ from the set $S_{k}^{b}$ and add add the nearest possible items of the lengts $s_{i+1}^{*}, \ldots, s_{i+l}^{*}$ into $S_{k}^{b}$ until the following condition

$s-\left(s_{1}^{*}+\ldots+s_{i-1}^{*}+s_{i+1}^{*}+\ldots+s_{i+l}^{*}\right)=r_{b}$ where $r_{b}<s_{l}$, $\iota=1,2, \ldots, n$ is satisfied and $S_{k}^{b}=\left[s_{1}^{*}, \ldots, s_{i-1}^{*}, s_{i+1}^{*}, \ldots, s_{i+1}^{*}\right]$.

If $r_{b}=0$ then $S_{c}:=S_{c} \cup\left\{S_{k}^{b}\right\}$ and go to 3 .

Else subtract $s_{j}^{*}$ from $S_{k}^{b}$ and add $s_{j+1}^{*}, \ldots, s_{j+l}^{*}$ into $S_{k}^{b}$ until the $s-\left(s_{1}^{*}+\ldots+s_{j-1}^{*}+s_{j+1}^{*}+\ldots+s_{j+l}^{*}\right)=r_{b}$ where $r_{b}<s_{l}$ is satisfied. If $S_{k}^{b}=S$ then $S_{c}:=S_{c} \cup\left\{S_{k}^{b}\right\}$ and $S_{c}$ represents the optimal solution, or optimal cuts of the rods. Else put

$$
R:=R \cup\left\{r_{b}\right], S^{*}:=S^{*} \cup\left\{S_{k}^{b}\right\}
$$

2. $b:=b-1$

If $b \geq 1$ go to 1 , else choose the minimal $r_{b}$ from the set $R$ and the relevant set $S_{k}^{b}$ from the set $S^{*}$.

3. Put $S:=\mathrm{S}-S_{k}^{b}, S_{c}:=S_{c} \cup\left\{S_{k}^{b}\right\}$.
If $S \neq \emptyset$ go to 0 .

Else, the family set $S_{c}$ represents the optimal cuts of the rods.

This algorithm was programmed in Matlab.

\section{Problems}

Practical realization was performed for several test problems. At first we present for illustration one simple problem below.

Problem 1. We have an unlimited number of rod stocks, each $600 \mathrm{~cm}$ length. The following 5 items must be cut (see the Table 1):

How is it possible to cut the rod stocks so that we minimize the waste?

Table 1

\begin{tabular}{|l|c|c|c|c|c|}
\hline Length of rods $(\mathrm{cm})$ & 55 & 42 & 27 & 22 & 16 \\
\hline Number of rods & 10 & 15 & 12 & 6 & 10 \\
\hline
\end{tabular}

Solution.

We present the solution of this problem by our algorithm in the following tables.

The Table 1.1 represents the solution of the first cut of the rods (the maximal length $s_{1}=55, b=10$ ). $S=[10 * 55,15 * 42,12 * 27$,

\begin{tabular}{|c|c|c|c|}
\hline \multicolumn{2}{|c|}{ 1. type 2. type 3. type 4. type 5. type } & $\sum$ & $\mathrm{rb}$ \\
\hline $10 * 55+1 * 42$ & & 592 & 8 \\
\hline $9 * 55 \quad+2 * 42$ & & 579 & 11 \\
\hline $8 * 55+3 * 42$ & $+1 * 27$ & 593 & 7 \\
\hline $7 * 55 \quad+5 * 42$ & & 595 & 5 \\
\hline $6 * 55 \quad+6 * 42$ & $+1 * 16$ & 598 & 2 \\
\hline $5 * 55+7 * 42$ & $+1 * 27$ & 596 & 4 \\
\hline $4 * 55+9 * 42$ & & 598 & 2 \\
\hline $3 * 55+10 * 42$ & & 585 & 15 \\
\hline $2 * 55+11 * 42$ & $+1 * 27$ & 599 & 1 \\
\hline $1 * 55+12 * 42$ & $+1 * 27$ & 586 & 14 \\
\hline
\end{tabular}
$6 * 22,10 * 16]((*)$-times $)$.

Table 1.1

The last but one row represents the best solution for the first cut. The minimal remainder $r_{2}=1$ and relevant $S_{1}^{2}=\{2 * 55$, $11 * 42,1 * 27\}$.

$$
S:=S-S_{1}^{2} \text {, i.e. } S=[8 * 55,4 * 42,11 * 27,6 * 22,10 * 16\}
$$

The Table 1.2 represents the solution of the second cut of the rods (the maximal length $s_{1}=55, b=8$ ). 
Table 1.2

\begin{tabular}{|lllll|c|c|}
\hline 1. type & 2. type & 3. type & 4. type & 5. type & $\sum$ & $r_{b}$ \\
\hline $8 * 55$ & $+3 * 42$ & $+1 * 27$ & $+1 * 16$ & 599 & 1 \\
\hline $7 * 55$ & $+4 * 42$ & $+1 * 27$ & $+1 * 16$ & 596 & 4 \\
\hline $6 * 55$ & $+4 * 42$ & $+3 * 27$ & $+1 * 16$ & 595 & 5 \\
\hline $\mathbf{5 * 5 5}$ & $+\mathbf{4} 4 \mathbf{4 2}$ & $+\mathbf{5} 27$ & $+\mathbf{1} \mathbf{2 2}$ & & $\mathbf{6 0 0}$ & $\mathbf{0}$ \\
\hline
\end{tabular}

The last row represents the best solution for the second cut. The minimal remainder $r_{2}=0$ and relevant $S_{2}^{5}=[5 * 55,4 * 42$, $5 * 27,1 * 22\}$

$$
S:=S-S_{2}^{5} \text {, i.e. } S=\{3 * 55,6 * 27,5 * 22,10 * 16\}
$$

The Table 1.3 represents the solution of the third cut of the rods (the maximal length $s_{1}=55, b=3$ ).

Table 1.3

\begin{tabular}{|c|c|c|c|c|c|}
\hline 1. type 2. type & 3. type & 4. type & 5. type & $\sum$ & $r_{b}$ \\
\hline $3 * 55$ & $+6 * 27$ & $+5 * 22$ & $+10 * 16$ & 597 & 3 \\
\hline
\end{tabular}

The remainder $r_{3}=3$ and $S_{3}^{3}=\{3 * 55,6 * 27,5 * 22,10 * 16\}$.

This row represents the last cut because $S:=S-S_{3}^{3}$, i.e. $S=\emptyset$.

Our optimal answer requires 3 rod stocks and has $r_{1}+r_{2}+$ $+r_{3}=1+0+3=4 \mathrm{~cm}$ waste. The summary of the solution is shown below in the next Table 2:

Table 2

\begin{tabular}{|l|c|c|c|c|c|c|}
\hline & \multicolumn{5}{|c|}{ Length of rods times number of rods } & sum \\
\hline 1-st rod stock & $55 * 2$ & $42 * 11$ & $27 * 1$ & & & 599 \\
\hline 2-nd rod stock & $55 * 5$ & $42 * 4$ & $27 * 5$ & $22 * 1$ & & 600 \\
\hline 3-rd rod stock & $55 * 3$ & & $27 * 6$ & $22 * 5$ & $16 * 10$ & 597 \\
\hline total & $55 * 10$ & $42 * 15$ & $27 * 12$ & $22 * 6$ & $16 * 10$ & 1796 \\
\hline
\end{tabular}

Imagine that we have one infinite rod stock and we need to cut this rod stock into the smaller rods mentioned above. Thus we need the total length of rod stock at least $T L=55^{*} 10+42 * 15+$ $+27 * 12+22 * 6+16^{*} 10=1796 \mathrm{~cm}$. This is the lower bound for the optimal solution.

Sum of three rod stocks is $1800 \mathrm{~cm}$. Our total length is $T=1796 \mathrm{~cm}$. The waste is $4 \mathrm{~cm}$. It is easy to see that our solution is minimal because our total length is equal to the lower bound, i.e. $T=T L$.

Problem 2. These next tasks arise from the practice. One business firm placed the special requirements. They needed to cut several rods of different lengths from rod stocks of different types and lengths.

We present the list of corresponding requirements in the Tables 3-7:

1-st requirement

Type: beam $840 \times 40 \mathrm{~L}$, length of rod stock $8000 \mathrm{~mm} \quad$ Table 3

\begin{tabular}{|l|c|c|c|c|c|c|c|}
\hline Length of rods $(\mathrm{mm})$ & 1450 & 1320 & 960 & 860 & 720 & 260 & 100 \\
\hline Number of rods & 60 & 3 & 80 & 30 & 12 & 30 & 30 \\
\hline
\end{tabular}

2-nd requirement

Type: beam $840 \times 40 \mathrm{~L}$, length of rod stock $8000 \mathrm{~mm} \quad$ Table 4

\begin{tabular}{|l|c|c|c|c|c|c|c|c|}
\hline Length of rods $(\mathrm{mm})$ & 1220 & 358 & 765 & 542 & 395 & 386 & 360 & 350 \\
\hline number of rods & 4 & 6 & 8 & 1 & 1 & 3 & 8 & 1 \\
\hline
\end{tabular}

\begin{tabular}{|l|c|c|c|c|c|c|c|c|}
\hline Length of rods (mm) & 345 & 305 & 300 & 290 & 260 & 250 & 170 & 100 \\
\hline number of rods & 1 & 1 & 1 & 4 & 4 & 2 & 14 & 6 \\
\hline
\end{tabular}

3-rd requirement

Type: tube D30, length of rod stock $6000 \mathrm{~mm}$ Table 5

\begin{tabular}{|l|c|c|c|}
\hline Length of rods (mm) & 1300 & 700 & 840 \\
\hline Number of rods & 6 & 33 & 81 \\
\hline
\end{tabular}

4-th requirement

Type: beam $520 \times 10$, length of rod stock $3000 \mathrm{~mm} \quad$ Table 6

\begin{tabular}{|l|c|c|c|c|c|c|}
\hline Length of rods $(\mathrm{mm})$ & 1400 & 1280 & 940 & 822 & 800 & 682 \\
\hline Number of rods & 1 & 1 & 15 & 4 & 6 & 2 \\
\hline
\end{tabular}

5 -th requirement

Type: beam of roller ledge, length of rod stock $3000 \mathrm{~mm} \quad$ Table 7

\begin{tabular}{|l|c|c|c|c|c|c|}
\hline Length of rods (mm) & 1400 & 1365 & 940 & 860 & 800 & 450 \\
\hline Number of rods & 4 & 4 & 20 & 2 & 8 & 2 \\
\hline
\end{tabular}

\section{Solutions of problems}

We present our answers for the all requirements mentioned above.

\section{Solution for the 1-st requirement}

The answer requires 27 rod stocks. It is presented in the following Table 8: 


\section{caMMNICOIIONS}

Table 8

\begin{tabular}{|l|c|c|c|c|c|c|c|c|c|c|c|c|c|c|}
\hline Ordinal number of the rod stock & 1 & 2 & 3 & 4 & 5 & 6 & 7 & 8 & 9 & 10 & 11 & 12 & 13 & 14 \\
\hline Remainder of the rod stock & 0 & 20 & 20 & 20 & 20 & 20 & 20 & 20 & 20 & 20 & 20 & 20 & 20 & 20 \\
\hline
\end{tabular}

\begin{tabular}{|l|c|c|c|c|c|c|c|c|c|c|c|c|c|}
\hline Ordinal number of the rod stock & 15 & 16 & 17 & 18 & 19 & 20 & 21 & 22 & 23 & 24 & 25 & 26 & 27 \\
\hline Remainder of the rod stock & 20 & 20 & 0 & 0 & 0 & 20 & 60 & 60 & 320 & 320 & 320 & 320 & 1280 \\
\hline
\end{tabular}

Our remainder is $O R=3000 \mathrm{~mm}$. The total length of the all rods is $T L=1450 * 60+1320 * 3+960 * 80+860 * 30+720 * 12+$ $+260 * 30+100 * 30=213000$. We had to use 27 rod stocks, i.e. $L=27 * 8000=216000 \mathrm{~mm}$.

The total remainder is $T R=L-T L=216000-213000=$ $=3000 \mathrm{~mm}$. The difference $\mathrm{D}$ of our remainder and the total remainder is $D=O R-T R=0 \mathrm{~mm}$. It means that we have found the best solution for this case.

\section{Solution for the 2-nd requirement}

Table 9

\begin{tabular}{|l|c|c|c|c|}
\hline Ordinal number of the rod stock & 1 & 2 & 3 & 4 \\
\hline Remainder of the rod stock & 0 & 5 & 40 & 3990 \\
\hline
\end{tabular}

Now the answer requires 4 rod stocks. Table 9 represents our solution together with the remainders on the every used rod stock.

Our remainder is $O R=4035 \mathrm{~mm}$. The total length of the all rods is $T L=1220 * 4+835 * 6+765 * 8+542 * 1+395 * 1+$ $+386 * 3+360 * 8+350 * 1+345 * 1+305 * 1+300 * 1+290 * 4+$ $+260 * 4+250 * 2+170 * 14+100 * 6=27965 \mathrm{~mm}$. It have been used 4 rod stocks, i.e. $L=4 * 8000=32000 \mathrm{~mm}$.

The total remainder is $T R=L-T L=32000-27965=$ $4035 \mathrm{~mm}$. The difference of our remainder and the total remainder is $D=O R-T R=0 \mathrm{~mm}$. We have found the best solution again.

\section{Solution for the 3-rd requirement}

The answer requires 17 rod stocks (see the Table 10 below):

Our remainder is $O R=3060 \mathrm{~mm}$. The total length of all the rods is $T L=1300 * 6+700 * 33+840 * 81=98940 \mathrm{~mm} .17 \mathrm{rod}$ stocks were used, i.e. $L=17 * 6000=102000 \mathrm{~mm}$.

The total remainder is $T R=L-T L=102000-98940=$ $=3060 \mathrm{~mm}$. The difference of our remainder and the total remainder is $D=O R-T R=0 \mathrm{~mm}$. As above we have again found the best solution.

\section{Solution for the 4-th requirement}

The answer to this requirement requires 10 rod stocks (see the Table 11):

Our remainder is $O R=3768 \mathrm{~mm}$. The total length of all the rods is $T L=1400 * 1+1280 * 1+940 * 15+822 * 4+800 * 6+$ $+682 * 2=26232 \mathrm{~mm}$. We have used 10 rod stocks. If we imagine that we have one infinite rod stock then the least integer multiple of 3000 greater than 26232 is 27000 for $n=9$, i.e. $L=9 * 3000$ $=27000 \mathrm{~mm}$.

The total remainder is $T R=L-T L=27000-26232=$ $=768 \mathrm{~mm}$. The difference of our remainder and the total remainder is $D=O R-T R=3000 \mathrm{~mm}$.

\section{Solution for the 5-th requirement}

The answer requires 14 rod stocks (see the Table 12):

Table 10

\begin{tabular}{|l|c|c|c|c|c|c|c|c|c|c|}
\hline Ordinal number of the rod stock & 1 & 2 & 3 & 4 & 5 & 6 & 7 & 8 & 9 \\
\hline Remainder of the rod stock & 40 & 40 & 40 & 120 & 120 & 120 & 120 & 120 & 120 \\
\hline Ordinal number of the rod stock & 10 & 11 & 12 & 13 & 14 & 15 & 16 & 17 \\
\hline Remainder of the rod stock & 120 & 120 & 120 & 120 & 120 & 120 & 400 & 1100 \\
\hline
\end{tabular}

Table 11

\begin{tabular}{|l|c|c|c|c|c|c|c|c|c|c|}
\hline Ordinal number of the rod stock & 1 & 2 & 3 & 4 & 5 & 6 & 7 & 8 & 9 & 10 \\
\hline Remainder of the rod stock & 98 & 180 & 180 & 180 & 180 & 298 & 96 & 556 & 600 & 1400 \\
\hline
\end{tabular}


Table 12

\begin{tabular}{|l|c|c|c|c|c|c|c|c|c|c|c|c|c|c|}
\hline Ordinal number of the rod stock & 1 & 2 & 3 & 4 & 5 & 6 & 7 & 8 & 9 & 10 & 11 & 12 & 13 & 14 \\
\hline Remainder of the rod stock & 180 & 180 & 180 & 180 & 180 & 180 & 200 & 200 & 30 & 10 & 35 & 35 & 270 & 1260 \\
\hline
\end{tabular}

Our remainder is $O R=3120 \mathrm{~mm}$. The total length of all the rods is $T L=1400 * 4+1365 * 4+940 * 20+860 * 2+800 * 8+$ $+450 * 2=38880 \mathrm{~mm}$. We used 14 rod stocks. There is an analogous reason as the one mentioned above, the ideal number of rod stocks is $n=13$, i.e. $L=13 * 3000=39000 \mathrm{~mm}$.

The total remainder is $T R=L-T L=39000-38880=$ $=120 \mathrm{~mm}$. The difference of our remainder and the total remainder is $D=O R-T R=3000 \mathrm{~mm}$.

\section{Evaluation}

In Problem 1 and in the first three tasks (requirements 1, 2, 3) of Problem 2 we obtained the exact solution, because our solution reached the lower bound of the requirement.

In the other tasks (requirements 4, 5) we used one more rod stock than the lower bound. Still these solutions can be accepted because the lower bound is the absolute minimum which does not need to be always reached. This is explained in the following example.

We have $3000 \mathrm{~mm}$ rod stocks and we need to cut 3 rods of the length $1600 \mathrm{~mm}$. It is obvious that we will use three $3000 \mathrm{~mm}$ rod stocks, because when one $1600 \mathrm{~mm}$ rod is cut, the remainder is $1400 \mathrm{~mm}$ and is useless. The lower bound is $3 * 1600=4800$ $\mathrm{mm}$, and $4800<6000 \mathrm{~mm}$, i.e. 2 rod stocks, we would only use $4800 \mathrm{~mm}$, which is less than two $3000 \mathrm{~mm}$ rod stocks. One less than three $3000 \mathrm{~mm}$ rod stocks.

The computational time of all the problems lasted under 1 second.

According to the facts and examples mentioned above it would be interesting to find exact solution and compare it with our solution. Let us point out that we were looking for the solution of the above Problem 1 by exact methodsmethods accessible on the internet http://code.google.com/p/cspsol/. Here the computational time is quite high, i.e. over one hour.

\section{Conclusion}

Our observations may be concluded as follows:

The longer the rod stocks are and the shorter our rods required are, the more closely we get to the lower bound, i.e. we can be sure about accuracy of our solution.

In practice we usually use the rod stocks of length 6000$8000 \mathrm{~mm}$. Using these lengths we reached exact solutions.

Supported by the grant KEGA 088 zU-4/2011.

\section{References}

[1] GILMORE, P. C., GOMORY, R. E.: A Linear Programming Approach to the Cutting-Stock Problem, Operation research 9, 1961, pp. 849-859.

[2] HAESSLER, R. W., SWEENEY, P. E.: Cutting Stock Problem and Solution Procedures, European J. of Operational Research 54, (1991), pp. 141-150.

[3] VICAN, J., GOCAL, J., JOST, J.: Fatigue Resistance of Typical Fatigue Prone Riveted Steel Railway Bridge Structural Detail, Communications - Scientific Letters of the University of Zilina, vol. 13, No. 3, 2011, pp. 5-8. 